\title{
Influence of two polyphenols on the structure and lubrication of salivary pellicle: An in vitro study on astringency mechanism
}

\author{
Lei LEI, Yue TANG, Jing ZHENG*, Genlei MA, Zhongrong ZHOU \\ Chengdu 610031, China \\ Received: 25 September 2020 / Revised: 23 December 2020 / Accepted: 18 January 2021 \\ (C) The author(s) 2021.
}

Tribology Research Institute, Key Laboratory of Advanced Technologies of Materials, Ministry of Education, Southwest Jiaotong University,

\begin{abstract}
This study investigated the influence of two polyphenols on the structure and lubrication of the salivary pellicle, aiming to extend the understanding of astringency mechanisms. The salivary pellicle was prepared by the adsorption of human whole saliva on the enamel substrate. Low-astringency catechin and high-astringency tannic acid were used as astringents. The changes induced by the two polyphenols in the structure and lubrication of the salivary pellicle were examined using quartz crystal microbalance with dissipation (QCM-D) and nanoindentation/scratch technique. The salivary pellicle suffers from changes in structure and physical properties owing to protein dehydration and protein-polyphenol complexation when encountering polyphenolic molecules, causing increases in the roughness and contact angle but a decrease in the load-bearing capacity. Therefore, the lubrication performance of the salivary pellicle is impaired, leading to an increase and fluctuation of the friction coefficient. The intensity of astringency has a strong positive correlation with the water contact angle, surface roughness, and friction coefficient of the salivary pellicle. In summary, astringency is a tactile perception driven by the roughness and wettability of the salivary pellicle rather than oral lubrication, and increased intraoral friction is an inevitable consequence of astringency. The findings of this study will help promote and assist the objective evaluation of astringency.
\end{abstract}

Keywords: astringency; salivary pellicle; structure; lubrication; polyphenol

\section{Introduction}

As a tactile perception, astringency is commonly defined as a drying, rough, and puckering mouthfeel after the consumption of foods containing polyphenols [1]. All solid substrata and mucosal membranes exposed to the oral environment are covered by a layer of absorbed salivary proteins, the so-called salivary pellicle [2]. The pellicle plays an extremely significant role in oral lubrication and tactile sensation [3]. Once introduced into the mouth, polyphenols, mainly tannic acid, interact with salivary proteins via hydrogen bonding and hydrophobic effects to form polyphenolprotein complexes and then alter the structure of the salivary pellicle and oral lubrication [4-6]. These changes are considered to be responsible for the increased activation of mechanoreceptors located within the mucosa, which in turn elicits astringency $[7,8]$.

Astringency has received a great amount of scientific interest, considering its potential application in both food nutriology and pharmacology [9]. Many previous studies have demonstrated that the occurrence of astringency is generally accompanied by increased intraoral friction. In vitro test results of Prinz and Lucas [10] showed that tannic acid causes a significant increase in intraoral friction because it precipitates salivary proteins and reduces the lubricating qualities of human saliva. Ma et al. [9] used mucoprotein as model protein to investigate the influence of tannic acid on saliva lubrication. They found that the

* Corresponding author: Jing ZHENG, E-mail: jzheng168@home.swjtu.edu.cn 
introduction of tannic acid increased the friction between a soft PDMS ball and the mucoproteinadsorbed glass surface and thus concluded that astringency is a consequence of protein-mediated lubrication failure. Brossard et al. [11] conducted human sensory tests and human saliva lubrication tests, and the results showed a strong positive correlation between the perceived intensity of astringency and the instrumental measured friction coefficient. Hence, many researchers have suggested that the astringency of polyphenol-rich products results at least initially from decreased oral lubrication, and it can be quantified using tribology techniques. However, different results came from Rossetti et al. [12]. They found that epicatechin, a polyphenol widely found in foods, did not alter the lubricating properties of human saliva, although it was perceived to be astringent. They pointed out that astringency is not a simple lubrication-driven tactile perception.

Oral lubrication mainly depends on the salivary pellicle, which has a thickness of 20-100 nm [13]. The mechanism of astringency is still beyond our understanding [7], but it has been widely accepted that polyphenols have the potential to alter the structure of the salivary pellicle through interactions with salivary proteins. Therefore, it can be deduced that the increased intraoral friction that occurs with astringency when encountering polyphenolic molecules, if present, may have resulted from the impaired lubrication performance of the salivary pellicle as a consequence of its structural change. Fully understanding the changes induced by polyphenolic molecules in the structure and physical properties of the salivary pellicle would help determine the role of saliva lubrication in astringency sensation and provide novel and useful insights into the origin of astringent mouthfeel and its objective evaluation. However, few studies have attempted to investigate how polyphenols alter the structure and physical properties of the salivary pellicle over time, although many efforts have been made to investigate the change in saliva lubrication during the development of astringency. The influence of polyphenols on the structure and physical properties of the salivary pellicle is still unclear.

In this study, the influence of two polyphenols commonly found in foods, catechin, and tannic acid, on the structure and lubrication performance of the salivary pellicle was investigated in vitro. The morphology, mechanical properties, and lubrication behavior of the salivary pellicle were characterized by atomic force microscopy (AFM) and nano-indentation/ scratch technique. The wettability of the salivary pellicle was evaluated using the contact angle. The change in the salivary pellicle structure over time was monitored using QCM-D. This study aimed to understand the manner in which polyphenolic molecules alter the structure and lubrication properties of the salivary pellicle and extend the understanding of astringency mechanisms.

\section{Materials and methods}

\subsection{Sample preparation}

Human whole saliva, collected from a single healthy, 25-year-old male donor using an unstimulated drool method in the morning [14-16], was used to prepare saliva samples. In order to avoid the degradation of salivary proteins caused by bacteria, the donor was asked to refrain from either eating or smoking before sampling $[17,18]$. The collected saliva was centrifuged at 2,000 $\mathrm{g}$ at $4{ }^{\circ} \mathrm{C}$ for $30 \mathrm{~min}$, and then the supernatant was extracted and used as saliva samples to perform tests within $2 \mathrm{~h}$.

Catechin and tannic acid aqueous solutions at a concentration of $1 \mathrm{~g} / \mathrm{L}$ were used as low-astringency and high-astringency polyphenol media, respectively.

Salivary pellicle samples were obtained by the adsorption treatment of the prepared saliva sample on a flat enamel substrate. Enamel substrates were prepared from freshly extracted human molars aged between 20 and 30 years. Each tooth was embedded into a denture base resin, and then ground and polished under constant water irrigation to obtain a flat enamel surface with average surface roughness $R_{\mathrm{a}}$ of not more than $0.02 \mu \mathrm{m}$ over a $1.0 \mathrm{~mm} \times 1.0 \mathrm{~mm}$ area [19]. During pellicle preparation, a drop of saliva sample was placed on the enamel substrate and left for $5 \mathrm{~min}$ at $25^{\circ} \mathrm{C}$, and then the residual saliva was drained carefully from the enamel surface using a dropper. The salivary pellicles obtained on the enamel substrates were divided into three groups. The first group, referred to as the original salivary pellicle, was used to perform tests without any treatment. The 
other two groups were treated with catechin or tannic acid before testing. During the polyphenol treatment, a drop of the previously prepared catechin or tannic acid aqueous solution was placed on the pellicle and removed after an 8-s interval. Thus, three salivary pellicle samples, original, catechin-treated, and tannic acid-treated, were prepared on enamel substrates. All pellicle samples were freshly prepared before testing.

\subsection{AFM characterizations}

The surface morphology and roughness of the freshly prepared salivary pellicle on the enamel substrate were characterized using AFM (Cypher, Oxford Instruments Asylum Research, UK) fitted with a silicon tip with a radius of $10 \mathrm{~nm}$ and in the tapping mode. The scan frequency was $1 \mathrm{~Hz}$, and the scan area was $10 \mu \mathrm{m} \times 10 \mu \mathrm{m}$. The pellicle was partly dehydrated during the testing process, but efforts were made both to shorten the drying time and to keep the testing time approximately the same for each sample.

\subsection{Nano-indentation/scratch tests}

The normal load-bearing capability of bio-films is generally evaluated by the penetration force. The thickness and penetration force of the salivary pellicle on the enamel substrate were measured through nano-indentation tests in the continuous stiffness measurement (CSM) mode. The tests were performed using a Berkovich diamond tip with a radius of $20 \mathrm{~nm}$ at a constant strain rate of $0.05 / \mathrm{s}$ and a limited thermal of $<0.05 \mathrm{~nm} / \mathrm{s}$ with a frequency of $45 \mathrm{~Hz}$ in a nanoindenter (G200, KLA-Tencor, USA). The maximum indentation depth was $500 \mathrm{~nm}$.

The lubrication behavior of the salivary pellicle on the enamel substrate was evaluated by unidirectional nano-scratch tests. Scratch tests were performed using a conical diamond tip with a radius of $10 \mu \mathrm{m}$ at a constant scratching speed of $10 \mu \mathrm{m} / \mathrm{s}$ under a normal load of $5 \mathrm{mN}$ in a nano-scratch tester (G200, KLATencor, USA). The scratch length was $100 \mu \mathrm{m}$.

Both indentation and scratch tests were conducted on freshly prepared salivary pellicle samples at $25^{\circ} \mathrm{C}$. Efforts were also made to keep the testing time approximately the same for each sample. For each pellicle, at least twelve independent measurements were conducted under each condition.

\subsection{Contact angle characterizations}

The wettability of the salivary pellicle on the enamel substrate was evaluated via the water contact angle. All contact angles were measured at $25{ }^{\circ} \mathrm{C}$ using a probe liquid of deionized water in a drop shape analyser system (DSA100, Krüss Corp., Germany). To obtain stable contact angles, the salivary pellicle samples were allowed to air dry at $25{ }^{\circ} \mathrm{C}$ for $1 \mathrm{~min}$ before testing, and the droplet was photographed after it rested on the sample surface for $20 \mathrm{~s}$ [18]. For each pellicle, the contact angle was determined through nine independent measurements.

\subsection{QCM-D measurements}

The change induced by either catechin or tannic acid in the structure of the salivary pellicle over time was measured by changes in the frequency $(\Delta f)$ and dissipation $(\Delta D)$ of a gold-coated quartz chip (Q-sense, QSX 301-standard gold, Sweden) using a QCM-D (Q-sense, Explorer, Sweden). A detailed description of the experimental setup and basic principles can be found elsewhere [20, 21].

QCM-D measurements were conducted at a flow rate of $80 \mu \mathrm{L} / \mathrm{min}$ at $25^{\circ} \mathrm{C}$. The baseline was recorded in deionized water. Each measurement included four stages (I, II, III, and IV). The measurement started with the injection of saliva into the measuring chamber (stage I). The flow was stopped after $15 \mathrm{~min}$ and was resumed during rinsing with deionized water (stage II). After rinsing for $5 \mathrm{~min}$, the previously prepared catechin or tannic acid aqueous solution was injected into the measuring chamber (stage III) for a period of $10 \mathrm{~min}$. Finally, deionized water was injected into the measuring chamber to rinse the chip (stage IV). The ending time of the four stages were referred to as $T_{I}$ $\mathrm{T}_{\mathrm{II}}, \mathrm{T}_{\mathrm{III}}$ and $\mathrm{T}_{\mathrm{IV}}$, respectively.

Some of the chips were taken off from the measuring chamber at either $\mathrm{T}_{\mathrm{II}}, \mathrm{T}_{\mathrm{III}}$, or $\mathrm{T}_{\mathrm{IV}}$, and the morphology of the salivary pellicle on the chip was characterized using scanning probe microscopy (SPM) with a scan frequency of $1 \mathrm{~Hz}$ in a nano-mechanical test system (TI 900, Hysitron Corp., USA). The pellicle was directly tested with SPM without any drying treatment. A globular tip with a radius of $1 \mu \mathrm{m}$ was used, and the scan area was $20 \mu \mathrm{m} \times 20 \mu \mathrm{m}$. 


\subsection{Statistical analysis}

The data are expressed as the mean \pm standard deviation in Section 3. They were analyzed by one-way analysis of variance with Tukey's test for multiple comparisons. The level of significance was set at a $P$-value $<0.01$.

\section{Results}

The results of AFM morphology characterizations using a $10 \mathrm{~nm}$ radius silicon tip demonstrated that polishing scratches are randomly scattered on the surface of the bare enamel. With the adsorption of the salivary pellicle consisting of a compact inner layer and evenly scattered small protuberances (generally called the outer layer), no scratches were observed on the enamel surface, while the surface roughness increased slightly (Fig. 1(a)). Obvious surface roughening occurred in the salivary pellicle after $8 \mathrm{~s}$

(a)

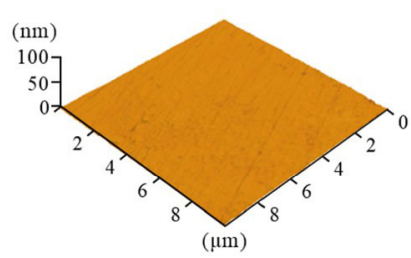

Catechin-treated

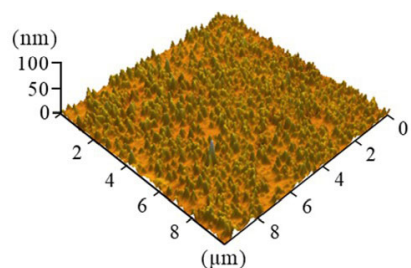

(b)

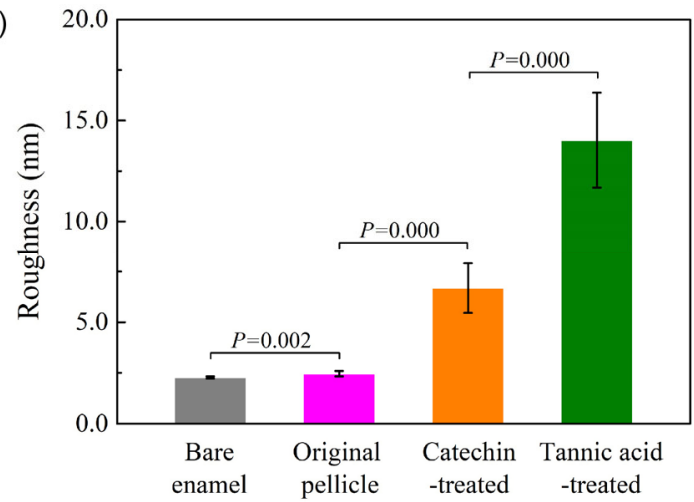

Fig. 1 (a) AFM morphologies and (b) roughnesses of salivary pellicles on enamel substrate subjected to different treatments. of catechin or tannic acid solution treatment. Imaging by AFM evinced a roughness increase of approximately $172 \%$ for the catechin and $471 \%$ for the tannic acid (Fig. 1(b)).

The interaction between salivary proteins and polyphenolic molecules such as catechin and tannic acid led to an increase in the thickness, but a decrease in the penetration force of the salivary pellicle; the results are illustrated in Fig. 2. Nano-indentation in the CSM mode indicated a thickness increase of approximately $19 \%$ for the catechin-treated pellicle and $52 \%$ for the tannic acid-treated pellicle. The penetration forces decreased by approximately $21 \%$ and $38 \%$, respectively.

As shown in Fig. 3, the bare enamel surface is hydrophilic, and its water contact angle was measured to be $63.9^{\circ}$. As a highly hydrated proteinaceous layer, the salivary pellicle on the enamel substrate makes the surface more hydrophilic, and the contact angle decreased to $16.2^{\circ}$. Polyphenol treatment leads to

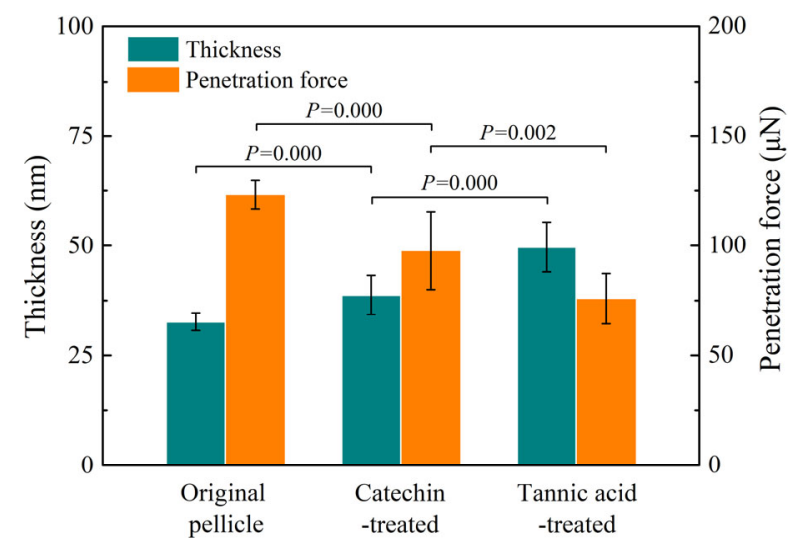

Fig. 2 Thickness and penetration force of salivary pellicles on enamel substrate subjected to different treatments.

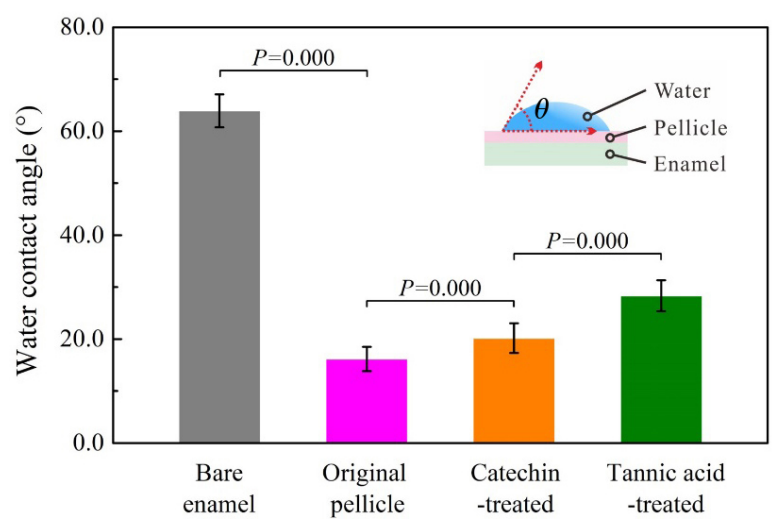

Fig. 3 Water contact angles of salivary pellicle on enamel substrates. 
an increase in the contact angle. The angle increased to $20.2^{\circ}$ and $28.4^{\circ}$ after the catechin and tannic acid treatments, respectively $(P$-value $<0.01)$.

The friction coefficients on enamel surfaces adsorbed with different salivary pellicles are shown in Fig. 4. The bare enamel surface was used as a control. The friction coefficient of the bare enamel surface exhibits slight fluctuation with a mean value of approximately 0.14 . The mean value and fluctuation of the friction coefficient decreased under the lubrication of the original salivary pellicle, but the coefficient increased and fluctuated significantly under the lubrication of the two polyphenol-treated pellicles, especially the tannic acid-treated pellicle. The mean friction coefficient under the lubrication of the tannic acid-treated pellicle, approximately 0.13 , was not significantly different to the bare enamel surface $(P$-value $<0.01)$, suggesting salivary pellicle lubrication failure.

The interaction between the salivary pellicle and the two polyphenol solutions was monitored by the
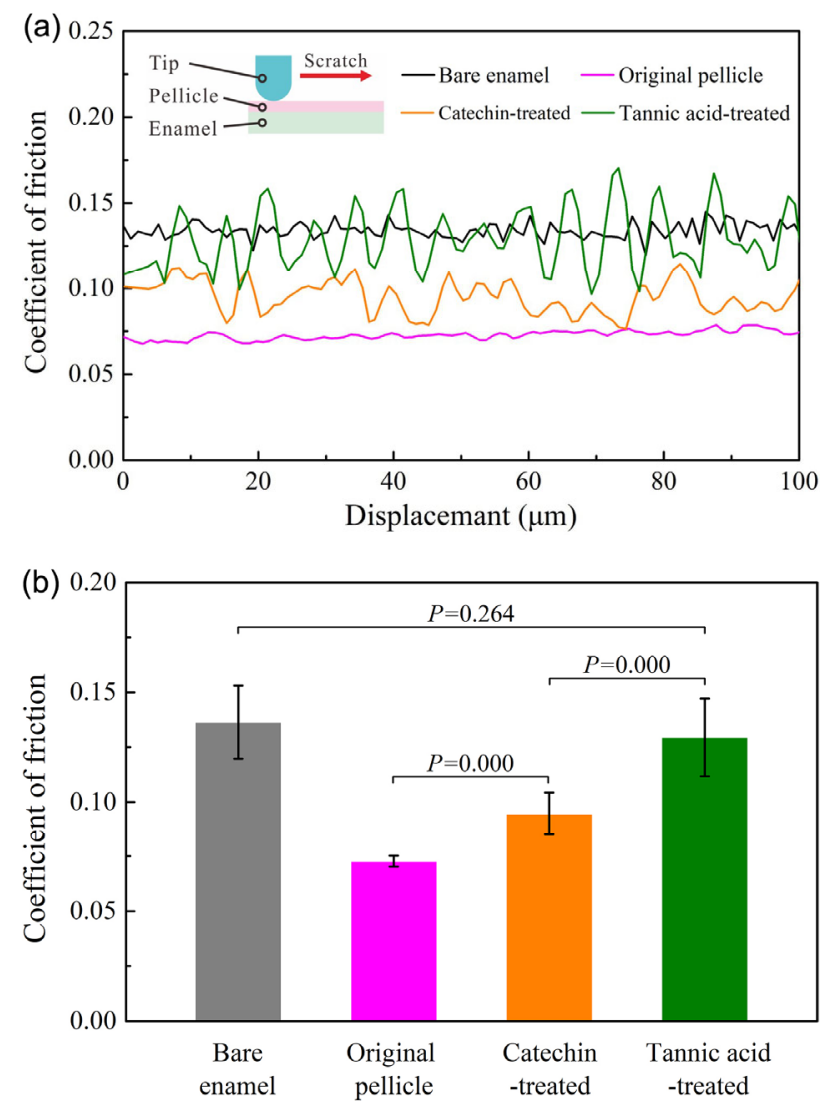

Fig. 4 Friction coefficients on enamel surfaces adsorbed with different salivary pellicles: (a) variation of friction coefficient with displacement and (b) mean friction coefficient. changes in frequency and dissipation of a gold-coated quartz chip using a QCM-D. As shown in Figs. 5(a) and $5(b)$, during stage $I$, the injection of saliva into the QCM-D chamber led to a rapid decrease in frequency and a rapid increase in dissipation, which means that a highly viscoelastic salivary pellicle was formed on the chip. An increase in frequency and a decrease in dissipation were detected during stage II of water rinsing, indicating the removal of some weakly adsorbed proteins from the outer layer of the pellicle. After sequentially injecting $1 \mathrm{~g} / \mathrm{L}$ catechin or tannic acid solution (stage III), the frequency slightly increased instantly and then decreased to a stable value, with the decrease using the tannic acid solution being more significant, which is attributed to the association of the salivary pellicle with polyphenolic molecules. Meanwhile, the dissipation increased rapidly to a stable value with the injection of catechin solution but decreased gradually to a stable value with the injection of tannic acid solution. After flushing with water to remove the free polyphenolic molecules (stage IV), an apparent increase in frequency along with a decrease in dissipation occurred in the catechintreated samples, while both the frequency and dissipation increased for the tannic acid-treated samples. Table 1 lists the thickness and $K$ values of the salivary pellicle on the chip at different stages calculated by the Q-Tools software with a Voigt model. The $K$ value represents the $\Delta D / \Delta f$ ratio, and it is positively correlated with film viscoelasticity [22].

SPM morphology examinations on the chip surface further confirmed the interaction between the salivary pellicle and polyphenol solutions. As shown in Fig. 5(c), a uniform and dense salivary pellicle appeared on the chip at $\mathrm{T}_{\mathrm{II}}$. The injection of both catechin and tannic acid solutions led to protein aggregation and even precipitation, and thus the protuberances in the outer layer of the pellicle look enlarged at $\mathrm{T}_{\mathrm{III}}$. The enlarged protuberances induced by catechin solution were mostly removed after sequentially rinsing with water to expose a dense pellicle on the chip at $T_{I V}$. The pellicle is obviously thinned, as shown in Table 1 . The protuberances induced by tannic acid solution are much larger and sparser, and they became short and obtuse after water rinsing. The thickness of the pellicle at $T_{\text {IV }}$ is only slightly smaller than that at $T_{\text {III }}$ (Table 1 ). 
(a)

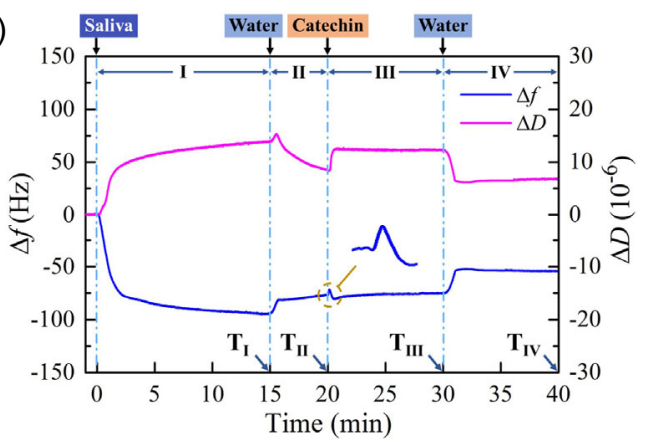

(c)
$\mathbf{T}_{\text {II }}$

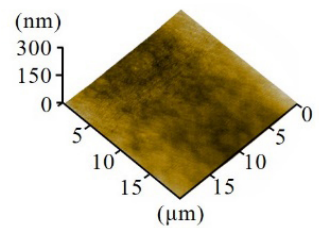

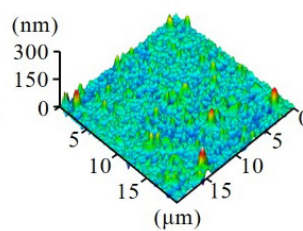

(b)

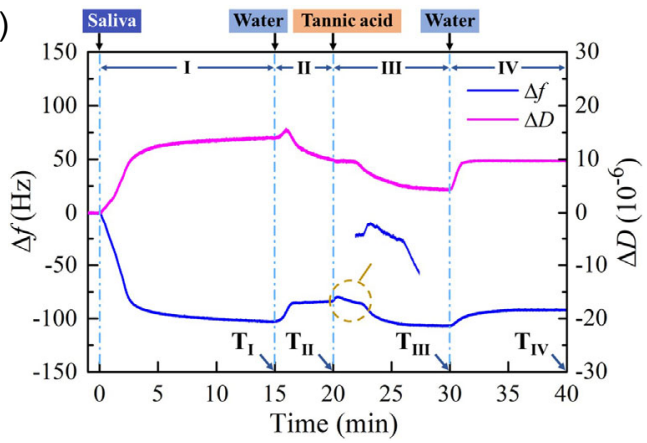

$\mathbf{T}_{\text {III }}$ $\mathbf{T}_{\mathrm{IV}}$
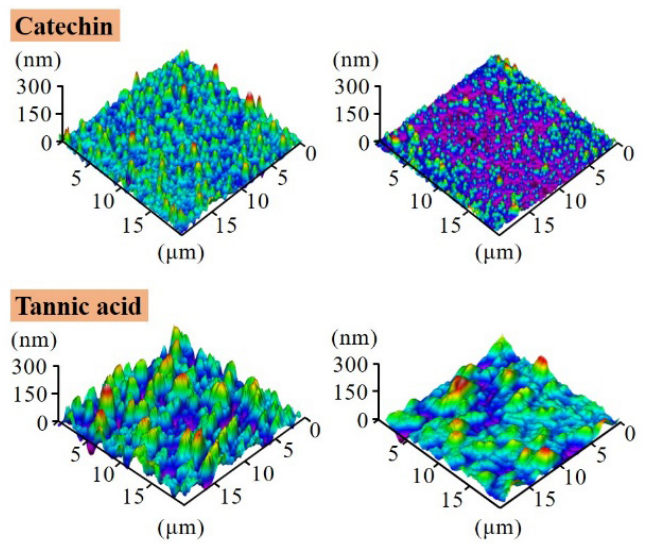

Fig. 5 Effects of (a) catechin and (b) tannic acid on the frequency and dissipation changes of adsorbed salivary pellicles determined by QCM-D, and (c) SPM morphologies captured on chip surface corresponding to the three time points of $\mathrm{T}_{\text {II }}, \mathrm{T}_{\mathrm{III}}$, and $\mathrm{T}_{\mathrm{IV}}$.

Table 1 Thickness and $K$ value of salivary pellicle on Au chip.

\begin{tabular}{|c|c|c|c|c|c|c|}
\hline \multirow{2}{*}{ Medium } & \multicolumn{3}{|c|}{ Thickness (nm) } & \multicolumn{3}{|c|}{$K\left(\times 10^{-7}\right)$} \\
\hline & $\mathrm{T}_{\text {II }}$ & $\mathrm{T}_{\text {III }}$ & $\mathrm{T}_{\mathrm{IV}}$ & $\mathrm{T}_{\text {II }}$ & $\mathrm{T}_{\text {III }}$ & $\mathrm{T}_{\mathrm{IV}}$ \\
\hline Catechin & $22.52 \pm 1.48$ & $23.23 \pm 1.22$ & $13.29 \pm 1.17$ & $1.13 \pm 0.07$ & $1.29 \pm 0.03$ & $1.03 \pm 0.02$ \\
\hline Tannic acid & $24.03 \pm 2.13$ & $31.98 \pm 3.17$ & $27.50 \pm 1.50$ & $1.10 \pm 0.11$ & $0.36 \pm 0.06$ & $1.03 \pm 0.11$ \\
\hline
\end{tabular}

\section{Discussion}

Saliva lubrication mainly depends on the salivary pellicle [23]. In this study, therefore, the influence of polyphenols on the structure and lubrication of the salivary pellicle was investigated, thereby revealing the relationship of intraoral friction to astringency and extending the understanding of the mechanisms of astringency sensation. How polyphenols alter the structure and physical properties of the salivary pellicle over time was investigated using QCM-D. Because of its high sensitivity, QCM-D is widely used in the structural analysis of biomembranes [24, 25]. Given that the salivary pellicle is about $20-100 \mathrm{~nm}$ thick [13], its mechanical and lubrication properties were characterized using the nano-indentation/scratch technique. Two polyphenols, low-molecular-weight catechin, and high-molecular-weight tannic acid, were used to fully understand the influence of polyphenols on the salivary pellicle.

In order to keep the surface energy as low as possible, the proteins in human saliva tend to be micelle-like. Hydrophobic amino acid side chains are packed in the interior, and outward hydrophilic amino acid side chains combine with water molecules. The hydrated protein micelles are adsorbed onto the surfaces of both teeth and oral soft tissues through selective physisorption to form a salivary pellicle with a multilayered structure in which the inner layer is much denser regardless of the substrata [26]. As shown in 
Figs. 1 and 2, after 8-s contact with $1 \mathrm{~g} / \mathrm{L}$ catechin or tannic acid solution, the small protuberances evenly scattered in the outer layer of the salivary pellicle become large and tend to be unevenly distributed, and the thickness and roughness of the pellicle increase, while the pellicle load-bearing capability decreases. In addition, the wettability of the pellicle decreases, which is manifested by an increase in the contact angle (Fig. 3). The changes caused by the tannic acid solution are much more significant. These observations suggest that the structure of the salivary pellicle changes due to the interaction of proteins with polyphenolic molecules. Real-time QCM-D measurements revealed the dynamic process of the change. As shown in Fig. 5, with the introduction of catechin or tannic acid solution (stage III), the frequency instantly increases slightly and then decreases to a stable value, and the decrease is greater than the increase. This means that the interaction of the salivary pellicle with polyphenol solutions experiences two stages: protein dehydration and then proteinpolyphenol complexation, causing a change in the pellicle structure. The changes in the frequency and dissipation caused by the injections of catechin and tannic acid solutions (stage III) and sequential water rinsing (stage IV) are different, which suggests that the interactions of catechin and tannic acid with salivary pellicle are different.

The interaction between polyphenolic molecules and proteins is mainly via hydrogen bonding between the hydroxyl groups of polyphenols and the carbonyl and amide groups of proteins, as well as the hydrophobic effect between the benzene rings of polyphenols and the non-polar amino acid side chains of proteins [27-29]. The hydrophobic effect contributes more to the interaction than hydrogen bonding. Figure 6 illustrates the schematic diagrams of the interactions of catechin and tannic acid with the salivary pellicle. Polyphenol molecules interact with proteins in the outer layer of the salivary pellicle. The affinity of polyphenols for proteins increases with polyphenol molecular weight because of the increased number of reaction sites. Low-molecular-weight polyphenols such as catechin have too few sites to form effective crosslinking with proteins, and they tend to form weak bonds with proteins [27, 30, 31]. Thus, catechin molecules mainly replace water molecules and combine with protein micelles via hydrogen bonding rather than unfolding the protein chains. The wettability of the salivary pellicle decreases as a result of protein dehydration, and the protein micelles in the outer layer of the pellicle are enlarged because catechin molecules are larger than water molecules. Pellicle thickness and roughness increase. However, the contact area among the proteins is decreased to weaken the bonding between them, since the proteins are bonded with each other via hydrogen bonding and van der Waals' forces [32]. This is supported by the increase in pellicle viscoelasticity (Table 1). Therefore, the outer proteins combined with catechin molecules were easily removed by water rinsing (Fig. 5(a)). Highmolecular-weight polyphenols such as tannic acid have enough sites to react with salivary proteins via hydrogen bonding and hydrophobic effects, resulting

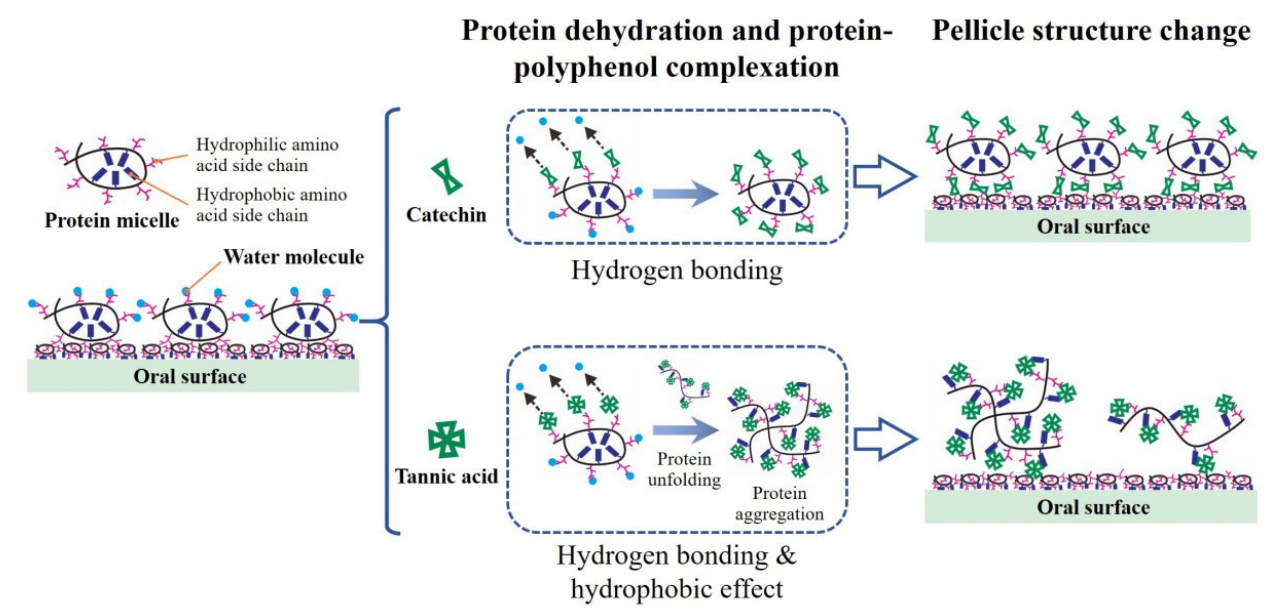

Fig. 6 Schematic diagram of the interactions of catechin and tannic acid with salivary pellicle. 
in unfolding of protein chains and protein aggregation. In other words, tannic acid molecules not only lead to protein dehydration but also form effective crosslinking with salivary proteins. The pellicle viscoelasticity decreased significantly (Table 1) because the molecular chain of tannic acid is rigid due to its high molecular weight. Compared with catechin, tannic acid has a more significant influence on the structure of the salivary pellicle, resulting in a more obvious decrease in the pellicle wettability and more significant increases in the pellicle thickness and roughness (Figs. 1-3). Subsequent water rinsing removes free tannic acid molecules rather than the protein agglomerates in the outer layer.

The roughness of the saliva pellicle with a multilayered structure is only about $2.5 \mathrm{~nm}$ (Fig. 1), and it has a certain load-bearing capacity, although it is only approximately $32.7 \mathrm{~nm}$ thick (Fig. 2). The pellicle effectively reduced the friction coefficient and its fluctuation on the enamel surface (Fig. 4), thereby showing good lubrication performance. The lubrication function of the salivary pellicle strongly depends on its structure $[33,34]$. Once the pellicle structure is destroyed, its mechanical properties deteriorate, causing salivary pellicle lubrication impairment and even failure. As shown in Figs. 1 and 2, when encountering catechin or tannic acid solutions, especially tannic acid solution, the pellicle thickness increases, but the load-bearing capacity of the pellicle decreases, and the roughness increases significantly. Hence, the value and fluctuation of the friction coefficient increased significantly under lubrication by the two polyphenoltreated pellicles (Fig. 4). There was no significant difference between the bare enamel surface and the lubrication of tannic acid-treated pellicle in the mean value of friction coefficient ( $P$-value $>0.01)$, and the latter fluctuates more obviously. This is the consequence of salivary pellicle lubrication failure caused by the significant effect of tannic acid molecules on the structure of the salivary pellicle. Therefore, it is inevitable that intraoral friction increases due to the impaired lubrication performance of the salivary pellicle because of its structural change when polyphenolic molecules are introduced into the mouth.

In general, oral surfaces innervated by tactile sensors are covered by the salivary pellicle [2,35], and oral-tactile sensations arise mainly from the changes induced by the consumed food and/or beverage in the integrity of the pellicle [3]. As mentioned above, astringency is commonly defined as a drying, rough, and puckering mouthfeel. Based on the results obtained in this study, it can be concluded that astringency is a tactile perception driven by salivary pellicle roughness and wettability rather than oral lubrication, and increased intraoral friction is an inevitable consequence of astringency. It is noteworthy that the salivary pellicle was regarded as a boundary lubricating layer in previous studies, and thus many researchers used macro-tribological tests to investigate the loss of saliva lubrication caused by polyphenolic compounds under the boundary lubrication regime [9]. As mentioned above, the salivary pellicle on the surfaces of both teeth and oral soft tissues, approximately 20-100 nm thick, has a stable multi-layer structure consisting of a uniform compact initial inner layer and outer layer with evenly scattered globular agglomerates $[26,36]$. Therefore, one possible mechanical function of the salivary pellicle in the mouth might be related to the form of thin film lubrication. The results of the present study demonstrate that different polyphenols have different effects on the lubricating properties of the saliva pellicle, and the effect of low-molecularweight polyphenols is weaker than that of highmolecular-weight polyphenols. The increase in intraoral friction induced by the polyphenols causing a slight impairment of salivary pellicle lubrication may be difficult to detect through macro-tribological tests. This might be the reason why Rossetti et al. [12] found that epicatechin, a polyphenol widely found in foods, did not alter the lubricating properties of human saliva.

Astringency is one of the most important qualities of many foods/beverages, such as red wines. At present, the astringency of red wines is evaluated through tasting by expert tasters, which inevitably involves a certain subjectivity [37]. An objective, reproducible, and easy-to-perform evaluation is of particular importance in the winemaking industry [38]. Many studies have focused on the correlation between astringency intensity and intraoral friction, aiming to quantify the astringency of polyphenol-rich products using tribology techniques. The results of the present study suggest that astringency is related to increases in the surface roughness and water contact angle of the salivary pellicle. Although both are astringent 
agents, the astringency intensity of tannic acid is greater than that of catechin [11]. Based on the humanperceived astringency intensities of catechin and tannic acid in a previous study and the results of this study (Table 2), the relationship between the astringency intensity and the water contact angle, roughness, and mean friction coefficient of the salivary pellicle was assessed using Pearson's correlation test, and the results are shown in Table 3. Pearson's correlation coefficient $(r=0.9969 ; P$-value $<0.05)$ showed a strong positive correlation between the astringency intensity and the water contact angle, roughness, and friction coefficient of the salivary pellicle. It seems that, as well as the friction coefficient, the water contact angle and surface roughness of the salivary pellicle can also be used for the objective evaluation of astringency. This would promote and assist the evaluation of astringency, considering that there are many factors that affect the measurement of intraoral friction [34, 39].

In this study, saliva was collected from one healthy donor. In order to avoid the influence of individual differences, a single donor was used to collect human whole saliva in many previous studies [14, 16, 40, 41]. The morphology, penetration force, and friction coefficient of the original salivary pellicle in Figs. 1, 2, and 4 are consistent with the results of previous studies $[23,42,43]$, suggesting that the saliva samples used in this study are representative. Given the fact that the drying process can induce changes in the pellicle, the salivary pellicle samples were freshly prepared and directly used for AFM, SPM, and nano-indentation/scratch tests without any drying treatment. Contact angle measurement was generally conducted on a dried salivary pellicle in previous studies [44, 45]. In this study, to minimize the effect of the drying process, the freshly prepared pellicle samples were only subjected to 1 min of air drying at $25{ }^{\circ} \mathrm{C}$ before contact angle measurement. In addition, in the present study, pellicle compositional analysis was not conducted, and the interaction between polyphenols and the salivary pellicle was monitored by the changes in frequency and dissipation of a gold-coated quartz chip using a QCM-D. Owing to its high sensitivity and real-time monitoring, QCM-D is a useful tool for the characterization of adsorption kinetics of thin films from proteins on surfaces and label-free analysis of biological molecules [9, 25, 46-48]. It should also be noted that the nano-indentation/ scratch test was conducted using an enamel substrate, while the QCM-D measurement was performed on gold-coated quartz chips. There are many types of standard chips used for QCM-D measurement, such as gold, silica, silver, and titanium chips. Gold-coated quartz chips are the most common among commercial QCM-D chips and have been used in previous studies concerning astringency $[9,48]$. Proteins may bind to the metallic surface and enamel surface via different bonds; thus, there may be some differences in the salivary pellicles obtained on gold-coated quartz chip surfaces and enamel surfaces. QCM-D was used to monitor the structural changes of the salivary pellicle induced by polyphenolic molecules in this study. As shown in Figs. 1(a) and 5(c), the salivary pellicle formed on gold-coated quartz chip surface also has a multi-layered structure, similar to that on the enamel surface, and the salivary pellicles on the two substrates experience similar structural changes when encountering polyphenolic molecules. The results

Table 2 Human-perceived astringency intensities of catechin and tannic acid [11] and corresponding water contact angle, mean friction coefficient, and roughness of salivary pellicle.

\begin{tabular}{ccccc}
\hline Medium & Astringency intensity & Pellicle contact angle $\left(^{\circ}\right)$ & Pellicle friction coefficient & $\begin{array}{c}\text { Pellicle surface } \\
\text { roughness (nm) }\end{array}$ \\
\hline Original pellicle & - & $16.16 \pm 2.34$ & $0.073 \pm 0.003$ & $2.46 \pm 0.14$ \\
Catechin & $4.36 \pm 2.70$ & $20.18 \pm 2.85$ & $0.095 \pm 0.010$ & $6.69 \pm 1.23$ \\
Tannic acid & $10.96 \pm 2.70$ & $28.36 \pm 2.98$ & $0.129 \pm 0.018$ & $14.03 \pm 2.35$ \\
\hline
\end{tabular}

Table 3 Pearson's correlation coefficients for astringency intensity with water contact angle, friction coefficient, and surface roughness of salivary pellicle.

\begin{tabular}{cccc}
\hline & Contact angle & Friction coefficient & Surface roughness \\
\hline Pearson's correlation coefficient & $0.99704^{\mathrm{a}}$ & $0.99998^{\mathrm{a}}$ & $0.99930^{\mathrm{a}}$ \\
\hline${ }^{\mathrm{a}}$ Correlation is significant at the 0.05 level (2-tailed). & &
\end{tabular}

${ }^{\mathrm{a}}$ Correlation is significant at the 0.05 level (2-tailed). 
suggest that gold-coated quartz chips are suitable for comparison of enamel surfaces.

Astringency has huge potential applications in both food nutriology and pharmacology [9]. Previous studies proposed various hypotheses to explain the mechanisms of the astringency sensation, but the exact mechanism is still unknown [11]. Here, we investigated the influence of two polyphenols on the structure and lubrication of the salivary pellicle. The results of this study suggest that the salivary pellicle on the oral surface suffers from changes in structure and physical properties as a consequence of protein dehydration and protein-polyphenol complexation when encountering polyphenolic molecules, causing increases in the pellicle roughness and contact angle but a decrease in the pellicle load-bearing capacity. As a result, the lubrication performance of the salivary pellicle is impaired, leading to increased intraoral friction. Astringency is a tactile perception driven by salivary pellicle roughness and wettability rather than oral lubrication, and the astringency intensity of polyphenolic compounds has a strong positive correlation with the water contact angle, surface roughness, and friction coefficient of the salivary pellicle. These findings extend the understanding of astringency mechanisms and help advance the objective evaluation of astringency. It should be noted that in the present study, only two polyphenols were used for research, and the adsorption treatment of saliva was implemented on the enamel substrate instead of on oral soft tissues. Previous studies reported that in the mouth, the proteins in saliva are adsorbed by physisorption onto teeth and oral soft tissues and form a salivary pellicle, and the pellicle is considered to have a multi-layered structure regardless of the substrata [26]. Our future studies will explore the influence of substrates on the salivary pellicle and the interaction of more polyphenolic compounds with the salivary pellicle.

\section{Conclusions}

The influence of two polyphenols, catechin, and tannic acid, on the structure and lubrication of the salivary pellicle have been studied using QCM-D and the nano-indentation/scratch technique in this study. The conclusions based on the given test conditions and results are summarized below:
1) The salivary pellicle suffers from changes in structure and physical properties as a consequence of protein dehydration and protein-polyphenol complexation when encountering polyphenolic molecules, causing increases in pellicle roughness and contact angle but a decrease in pellicle load-bearing capacity. As a result, the lubrication performance of the salivary pellicle is impaired, leading to an increase and fluctuation of the friction coefficient.

2) Astringency is a tactile perception driven by salivary pellicle roughness and wettability rather than oral lubrication, and increased intraoral friction is an inevitable consequence of astringency.

3) The astringency intensity of polyphenolic compounds has a strong positive correlation with the water contact angle, surface roughness, and friction coefficient of the salivary pellicle. The astringency of polyphenol-rich products can be quantified by means of the water contact angle and surface roughness of the salivary pellicle.

\section{Acknowledgements}

This work was supported by the National Natural Science Foundation of China (51675449 and 51535010), the National Defense Science and Technology Key Laboratory Fund (614220206021802), and the 111 Project (B20008).

Open Access This article is licensed under a Creative Commons Attribution 4.0 International License, which permits use, sharing, adaptation, distribution and reproduction in any medium or format, as long as you give appropriate credit to the original author(s) and the source, provide a link to the Creative Commons licence, and indicate if changes were made.

The images or other third party material in this article are included in the article's Creative Commons licence, unless indicated otherwise in a credit line to the material. If material is not included in the article's Creative Commons licence and your intended use is not permitted by statutory regulation or exceeds the permitted use, you will need to obtain permission directly from the copyright holder.

To view a copy of this licence, visit http://creativecommons.org/licenses/by/4.0/. 


\section{References}

[1] Mcrae J M, Falconer R J, Kennedy J A. Thermodynamics of grape and wine tannin interaction with polyproline: Implications for red wine astringency. J Agric Food Chem 58(23): 12510-12518 (2010)

[2] Hannig M. The protective nature of the salivary pellicle. Int Dent J 52(S5): 417-423 (2002)

[3] Laguna L, Sarkar A, Bryant M G, Beadling A R, Bartolomé B, Victoria Moreno-Arribas M. Exploring mouthfeel in model wines: Sensory-to-instrumental approaches. Food Res Int 102: 478-486 (2017)

[4] Gawel R. Red wine astringency: A review. Aust J Grape Wine Res 4(2): 74-95 (1998)

[5] Joslyn M A, Goldstein J L. Astringency of fruits and fruit products in relation to phenolic content. Adv Food Res 13: 179-217 (1964)

[6] Singleton V L, Noble A C. Wine flavor and phenolic substances. In Phenolic, Sulfur, and Nitrogen Compounds in Food Flavors. Charalambous G, Katz I, Eds. Washington, D. C. : American Chemical Society, 1976: 48-49.

[7] Chen J S. Food oral processing: Some important underpinning principles of eating and sensory perception. Food Struct 1(2): 91-105 (2014)

[8] Gibbins H L, Carpenter G H. Alternative mechanisms of astringency - what is the role of saliva? J Texture Stud 44(5): 364-375 (2013)

[9] Ma S H, Lee H, Liang Y M, Zhou F. Astringent mouthfeel as a consequence of lubrication failure. Angew Chem Int Ed Engl 55(19): 5793-5797 (2016)

[10] Prinz J F, Lucas P W. Saliva tannin interactions. J Oral Rehabilitation 27(11): 991-994 (2008)

[11] Brossard N, Cai H F, Osorio F, Bordeu E, Chen J S. "Oral" tribological study on the astringency sensation of red wines. J Texture Stud 47(5): 392-402 (2016)

[12] Rossetti D, Bongaerts J H H, Wantling E, Stokes J R, Williamson A M. Astringency of tea catechins: More than an oral lubrication tactile percept. Food Hydrocoll 23(7): 1984-1992 (2009)

[13] Lendenmann U, Grogan J, Oppenheim F G. Saliva and dental pellicle: A review. Adv Dent Res 14: 22-28 (2000)

[14] Sipahi C, Anil N, Bayramli E. The effect of acquired salivary pellicle on the surface free energy and wettability of different denture base materials. J Dent 29(3): 197-204 (2001)

[15] Munro C L, Grap M J, Jablonski R, Boyle A. Oral health measurement in nursing research: State of the science. Biol Res Nurs 8(1): 35-42 (2006)

[16] Wetton S, Hughes J, West N, Addy M. Exposure time of enamel and dentine to saliva for protection against erosion: A study in vitro. Caries Res 40(3): 213-217 (2006)

[17] Chiappin S, Antonelli G, Gatti R, De Palo E F. Saliva specimen: A new laboratory tool for diagnostic and basic investigation. Clin Chim Acta 383(1-2): 30-40 (2007)

[18] Zhang Y F, Zheng J, Zheng L, Zhou Z R. Effect of adsorption time on the adhesion strength between salivary pellicle and human tooth enamel. J Mech Behav Biomed Mater 42: 257-266 (2015)

[19] Xiao H, Lei L, Peng J P, Yang D, Zeng Q H, Zheng J, Zhou $\mathrm{Z}$ R. Research of the role of microstructure in the wear mechanism of canine and bovine enamel. J Mech Behav Biomed Mater 92: 33-39 (2019)

[20] Barrantes A, Arnebrant T, Lindh L. Characteristics of saliva films adsorbed onto different dental materials studied by QCM-D. Colloids Surfaces A: Physicochem Eng Aspects 442: 56-62 (2014)

[21] Rodahl M, Höök F, Krozer A, Brzezinski P, Kasemo B. Quartz crystal microbalance setup for frequency and Q-factor measurements in gaseous and liquid environments. Rev Sci Instrum 66(7): 3924-3930 (1995)

[22] Höök F, Rodahl M, Kasemo B, Brzezinski P. Structural changes in hemoglobin during adsorption to solid surfaces: Effects of $\mathrm{pH}$, ionic strength, and ligand binding. PNAS 95(21): 12271-12276 (1998)

[23] Zeng Q H, Ma G L, Xiao H, Yang D, Zheng J, Zheng L, Zhou Z R. Effect of saliva flow rate on the adsorption kinetics and lubrication of salivary pellicle on human tooth enamel surface. Wear 426-427: 180-185 (2019)

[24] Macakova L, Yakubov G E, Plunkett M A, Stokes J R. Influence of ionic strength changes on the structure of pre-adsorbed salivary films. A response of a natural multicomponent layer. Colloids Surf B 77(1): 31-39 (2010)

[25] Santos O, Lindh L, Halthur T, Arnebrant T. Adsorption from saliva to silica and hydroxyapatite surfaces and elution of salivary films by SDS and delmopinol. Biofouling 26(6): 697-710 (2010)

[26] Ash A, Burnett G R, Parker R, Ridout M J, Rigby N M, Wilde P J. Structural characterisation of parotid and whole mouth salivary pellicles adsorbed onto DPI and QCMD hydroxyapatite sensors. Colloids Surf B Biointerfaces 116: 603-611 (2014)

[27] Charlton A J, Baxter N J, Khan M L, Moir A J G, Haslam E, Davies A P, Williamson M P. Polyphenol/peptide binding and precipitation. J Agric Food Chem 50(6): 1593-1601 (2002)

[28] Santos-Buelga C, Freitas V D. Influence of phenolics on wine organoleptic properties. In Wine Chemistry and Biochemistry. Moreno-Arribas M V, Polo M C, Eds. New York: Springer, 2009: 551-553.

[29] Poncet-Legrand C, Cartalade D, Putaux J L, Cheynier V, Vernhet A. Flavan-3-ol aggregation in model ethanolic solutions: incidence of polyphenol structure, concentration ethanol content and ionic strength. Langmuir 19(25): 1056310572 (2003) 
[30] de Freitas U V, Mateus U N. Protein/polyphenol interactions: Past and present contributions. mechanisms of astringency perception. Curr Org Chem 16(6): 724-746 (2012)

[31] Hagerman A E, Rice M E, Ritchard N T. Mechanisms of protein precipitation for two tannins, pentagalloyl glucose and epicatechin16 $(4 \rightarrow 8)$ catechin (procyanidin). J Agric Food Chem 46(7): 2590-2595 (1998)

[32] Zhang Y F, Zheng J, Zheng L, Shi X Y, Qian L M, Zhou Z R. Effect of adsorption time on the lubricating properties of the salivary pellicle on human tooth enamel. Wear 301(1-2): 300-307 (2013)

[33] Yakubov G E, Macakova L, Wilson S, Windust J H C, Stokes J R. Aqueous lubrication by fractionated salivary proteins: Synergistic interaction of mucin polymer brush with low molecular weight macromolecules. Tribol Int 89: 34-45 (2015)

[34] Zeng Q H, Zheng L, Zhou J, Xiao H, Zheng J, Zhou Z R. Effect of alcohol stimulation on salivary pellicle formation on human tooth enamel surface and its lubricating performance. J Mech Behav Biomed Mater 75: 567-573 (2017)

[35] Laguna L, Bartolomé B, Moreno-Arribas M V. Mouthfeel perception of wine: Oral physiology, components and instrumental characterization. Trends Food Sci Technol 59: 49-59 (2017)

[36] Hannig M. Ultrastructural investigation of pellicle morphogenesis at two different intraoral sites during a 24-h period. Clin Oral Investig 3(2): 88-95 (1999)

[37] Valentová H, Skrovánková S, Panovská Z, Pokorný J. Time-intensity studies of astringent taste. Food Chem 78(1): 29-37 (2002)

[38] Sarneckis C J, Dambergs R G, Jones P, Mercurio M, Herderich M J, Smith P A. Quantification of condensed tannins by precipitation with methyl cellulose: Development and validation of an optimised tool for grape and wine analysis. Aust J Grape Wine Res 12(1): 39-49 (2006)

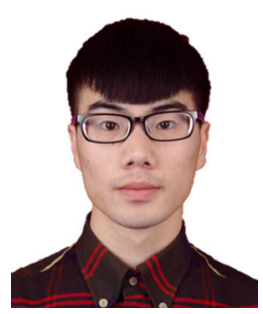

Lei LEI. He received his B.S. degree in mechanical engineering in 2015 from Heilongjiang University of Science and Technology, Harbin, China. After then, he was a master

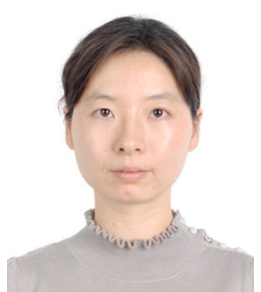

Jing ZHENG. She received her Ph.D. degree in mechanical engineering in 2005 from the Southwest Jiaotong University, Chengdu, China. She joined the Tribology Research
[39] Hahn Berg I C, Lindh L, Arnebrant T. Intraoral lubrication of PRP-1, statherin and mucin as studied by AFM. Biofouling 20(1): 65-70 (2004)

[40] Arvidsson A, Lofgren C D, Christersson C E, Glantz P O, Wennerberg A. Characterisation of structures in salivary secretion film formation. An experimental study with atomic force microscopy. Biofouling 20(3): 181-188 (2004)

[41] Hahnel S, Wieser A, Lang R, Rosentritt M. Biofilm formation on the surface of modern implant abutment materials. Clin Oral Implants Res 26(11): 1297-1301 (2015)

[42] Dickinson M E, Mann A B. Nanomechanics and morphology of salivary pellicle. J Mater Res 21(8): 1996-2002 (2006)

[43] Zeng Q, Zheng J, Yang D, Tang Y, Zhou Z. Effect of calcium ions on the adsorption and lubrication behavior of salivary proteins on human tooth enamel surface. $J$ Mech Behav Biomed Mater 98: 172-178 (2019)

[44] Morge S, Adamczak E, Lindén L Å. Variation in human salivary pellicle formation on biomaterials during the day. Arch Oral Biol 34(8): 669-674 (1989)

[45] van der Mei H C, White D J, Kamminga-Rasker H J, Knight J, Baig A A, Smit J, Busscher H J. Influence of dentifrices and dietary components in saliva on wettability of pelliclecoated enamel in vitro and in vivo. Eur J Oral Sci 110(6): 434-438 (2002)

[46] Yao J W, Xiao Y, Lin F. Effect of various $\mathrm{pH}$ values, ionic strength, and temperature on papain hydrolysis of salivary film. Eur J Oral Sci 120(2): 140-146 (2012)

[47] Ash A, Mulholland F, Burnett G R, Wilde P J. Structural and compositional changes in the salivary pellicle induced upon exposure to SDS and STP. Biofouling 30(10): 1183-1197 (2014)

[48] Guerreiro J R L, Teixeira N, De Freitas V, Sales M G F, Sutherland D S. A saliva molecular imprinted localized surface plasmon resonance biosensor for wine astringency estimation. Food Chem 233: 457-466 (2017)

student in the Tribology Research Institute at Southwest Jiaotong University, Chengdu, China. He became a Ph.D. student at the same university. His research interests include the tribology of natural teeth, biolubrication, and bionic tribology.

Institute at Southwest Jiaotong University in 2000. Her current position is a professor. Her research areas cover the tribology of natural teeth and dental materials, bio-lubrication, and bionic tribology. 